\title{
The optimal timing of continuous renal replacement therapy according to the modified RIFLE classification in critically ill patients with acute kidney injury: a retrospective observational study
}

\author{
Jun Suzuki ${ }^{1}$, Tetsu Ohnuma², Hidenori Sanayama ${ }^{3}$, Kiyonori Ito ${ }^{4}$, Takayuki Fujiwara ${ }^{5}$, Hodaka Yamada ${ }^{6}$
} Alan Kawarai Lefor ${ }^{7}$ and Masamitsu Sanui ${ }^{2^{*}}$ (D)

\begin{abstract}
Background: Acute kidney injury (AKI) requiring continuous renal replacement therapy (CRRT) is associated with high mortality in critically ill patients. However, the optimal timing to initiate CRRT in patients with AKI is unknown. The purpose of this study is to investigate whether the timing of initiation of CRRT according to severity of AKI is associated with in-hospital mortality.

Methods: We retrospectively reviewed 189 patients treated with CRRT for AKI in the intensive care unit between January 2009 and February 2013. Patients aged <18 years or receiving renal replacement therapy for end-stage renal disease were excluded. The modified RIFLE classification was used to stratify patients into two groups at initiation of CRRT, including early (no AKI or risk) and late (injury or failure).
\end{abstract}

Results: There were 52 (28\%) patients in the early group and 137 (72\%) patients in the late group. The median age was 72 (range 61-78) years, including 70\% males. The median intensive care unit and hospital stays were 10 (4-18) and 26 (13-58) days, respectively. Crude early vs. late group intensive care unit mortality was 50 vs. 44\% ( $P=0.51)$, and in-hospital mortality was 64 vs. $50 \%(P=0.10)$, respectively. Logistic regression analysis showed that late initiation $(\mathrm{OR}, 0.30 ; 95 \% \mathrm{Cl}, 0.13-0.71 ; P=0.006)$ and lower SAPS score $(\mathrm{OR}, 1.04 ; 95 \% \mathrm{Cl}, 1.02-1.06 ; P<0.001)$ were independently associated with decreased mortality.

Conclusions: This study suggests that late initiation of CRRT is associated with a lower risk of in-hospital mortality in patients with AKI. Further studies are needed to confirm the optimal timing for initiation of CRRT.

Keywords: Acute kidney injury, Continuous renal replacement therapy, Timing, RIFLE classification

\section{Background}

Acute kidney injury (AKI) is a life-threatening complication with an incidence of $36-67 \%$ in critically ill patients [1, 2]. Approximately $4-5 \%$ of patients with AKI in the intensive care unit (ICU) require continuous renal replacement therapy (CRRT), resulting in a mortality rate greater than $50 \%[3,4]$.

\footnotetext{
* Correspondence: msanui@mac.com

${ }^{2}$ Department of Anesthesiology and Critical Care Medicine, Jichi Medical University Saitama Medical Center, 1-847 Amanuma, Omiya-ku, Saitama City, Saitama 330-8503, Japan

Full list of author information is available at the end of the article
}

Although a consensus does not exist for the timing of initiation of renal replacement therapy (RRT) in patients with AKI, early initiation of RRT tended to improve mortality. A recent systematic review and meta-analysis concluded that early RRT initiation in critically ill patients with AKI may result in a survival benefit [5]. However, the study included only two small randomized clinical trials evaluating the timing of RRT initiation, and the definitions of classification of AKI and RRT initiation as "early" or "late" RRT varied significantly between the studies. Although two recent prospective 
randomized trials were reported [6,7], the results were conflicting.

The RIFLE classification, developed by the Acute Dialysis Quality Initiative in 2004, classifies AKI patients into five groups according to creatinine and urine output criteria. The groups include Risk, Injury, Failure, Loss, and End-stage renal disease [8]. This classification was reported to be a useful system for predicting AKI severity and in-hospital mortality in patients with AKI $[1,9,10]$. Recent retrospective and prospective observational studies used the modified RIFLE classification as a marker for the optimal timing of RRT initiation for postoperative or septic patients with AKI [11-14].

The aim of this study was to evaluate whether early or late initiation of CRRT defined by the modified RIFLE classification is associated with in-hospital mortality in medical and surgical critically ill patients with AKI. We hypothesized that early initiation of CRRT is associated with lower in-hospital mortality than the late initiation.

\section{Methods}

\section{Study population}

We retrospectively reviewed the records of patients admitted to the combined medical-surgical intensive care unit (ICU) at Jichi Medical University Saitama Medical Center between January 2009 and February 2013. This study was approved by the Institutional Review Board of Jichi Medical University Saitama Medical Center. Patients aged less than 18 years with end-stage renal disease treated with chronic hemodialysis were excluded. If multiple CRRT treatments were performed on one patient in the ICU, only the first CRRT treatment was considered in this analysis. The diagnosis of AKI was made according to the modified RIFLE classification (Table 1) defined using only estimated glomerular filtration rate (eGFR) criteria comparing pre-admission baseline serum creatinine levels with those at the time of initiating CRRT. If the pre-admission baseline creatinine was unknown, a baseline eGFR of $75 \mathrm{ml} / \mathrm{min} / 1.73 \mathrm{~cm}^{2}$ was used based on a calculation from the abbreviated Modification of Diet in Renal Disease, as suggested by the Acute Dialysis Quality Initiative [15].

Table 1 Categorization of patients based on the Modified RIFLE classification $(n=189)$

\begin{tabular}{lll}
\hline Modified RIFLE classification & eGFR criteria (\%) & $n(\%)$ \\
\hline Non-acute kidney injury & eGFR decrease $\leq 25$ & $16(9)$ \\
Risk & eGFR decrease $>25$ & $36(19)$ \\
Injury & eGFR decrease $>50$ & $70(37)$ \\
Failure & eGFR decrease $>75$ & $67(35)$ \\
\hline
\end{tabular}

Abbreviations: eGFR estimated glomerular filtration rate

\section{Data collection}

Chart reviews including baseline characteristics and laboratory data were performed in eligible patients. The characteristics reviewed included age, gender, primary diagnosis, creatinine at baseline and at ICU admission, and pre-existing comorbidities, including hypertension, diabetes mellitus, chronic kidney disease (CKD), and congestive heart failure. CKD is defined as an eGFR less than $60 \mathrm{ml} / \mathrm{min} / 1.73 \mathrm{~cm}^{2}$ over 3 months or previously diagnosed. CKD is classified into five groups defined by the CKD guidelines [16]. The Simplified Acute Physiology Score (SAPS) II was calculated on the day of ICU admission [17]. We recorded the use of mechanical ventilation, vasopressors, diuretics, $\mathrm{PaO}_{2} / \mathrm{FiO}_{2}$ ratio, Glasgow Coma Scale, bilirubin, platelets, albumin, urine output, creatinine, eGFR, and blood urea nitrogen (BUN) at the time of CRRT initiation. Continuous venovenous hemodiafiltration was used in all patients. We used dialysis membranes composed of polysulfone, polymethylmethacrylate, and polyacrylonitrile. Continuous veno-venous hemodiafiltration was performed with blood flow rates ranging from 80 to $120 \mathrm{ml} / \mathrm{min}$, a dialysis flow rate ranging from 500 to $3950 \mathrm{ml} / \mathrm{h}$, and a replacement flow rate ranging from 0 to $3800 \mathrm{ml} / \mathrm{h}$.

The following general indications for RRT were used: (1) metabolic acidosis ( $\mathrm{pH}<7.2$ and serum bicarbonate $<16 \mathrm{mEq} / \mathrm{l}$ in arterial blood gas), (2) hyperkalemia (serum $\left.\mathrm{K}^{+}>5.5 \mathrm{mmol} / \mathrm{l}\right)$, (3) azotemia (BUN $>150 \mathrm{mg} / \mathrm{dl}$ ) with uremic symptoms, (4) oliguria/anuria (urine output $<100 \mathrm{ml} / 12 \mathrm{~h}$ ), or otherwise (5) the indications were at the discretion of the treating physician.

Patients were categorized into four groups (non-AKI, risk, injury, and failure) according to the modified RIFLE classification (Table 1). Early initiation was defined as initiation of CRRT in patients in the non-AKI or risk groups, whereas patients classified in the injury or failure group constituted the late initiation group (8). Factors contributing to AKI were selected from a list of the following choices: septic shock, cardiogenic shock, drug-induced AKI, hypovolemia, major surgery, and others (4). In each case, one or more contributing factors could be selected. Sepsis was defined based on systemic inflammation syndrome and consensus guidelines [18].

As a primary outcome, we compared in-hospital mortality between the groups. We also reviewed the duration of CRRT, ICU length of stay, post-RRT hospital length of stay, hospital length of stay, time interval from ICU admission to RRT initiation, and ICU mortality as secondary outcomes.

\section{Statistical analysis}

Data are presented as medians and interquartile ranges (25th to 75th percentiles) or percentages, as appropriate. 
The chi-square test or Fisher's exact test were used for nominal variables and the Mann-Whitney test was used for continuous variables. A $P$ value $<0.05$ was considered statistically significant. Single variables with a $P$ value of less than 0.12 were then applied into multivariable logistic regression analysis, which was performed to evaluate risk factors for hospital mortality. The following variables were included as covariates in a logistic regression analysis: age, gender, hypertension, CKD, SAPS II, vasopressor use, mechanical ventilation use, hypovolemia, $\mathrm{PaO}_{2} / \mathrm{FiO}_{2}$ ratio at CRRT initiation, urine output at CRRT initiation, BUN at CRRT initiation, albumin at CRRT initiation, and early vs. late CRRT initiation. All statistical analyses were performed with EZR which is a graphical user interface for $\mathrm{R}$ (The $\mathrm{R}$ Foundation for Statistical Computing, Vienna, Austria, version 2.13.0) [19]. EZR is a modified version of the $R$ commander designed to add statistical functions frequently used in biostatistics.

\section{Results}

A total of 189 patients were included in this analysis. There were 16 patients (9\%) in the non-AKI group, 36
(19\%) in the risk group, $70(37 \%)$ in the injury group, and $67(35 \%)$ in the failure group, according to the modified RIFLE classification (Table 1). In the early and late CRRT initiation groups, there were $52(28 \%)$ and 137 patients (72\%), respectively.

Baseline characteristics and comorbidities are shown in Table 2. The median age was $72(61-78)$ years, and $70 \%$ were male. Pre-admission baseline creatinine was available for 124 (66\%) patients, and the median creatinine was $1.0(0.7-1.5) \mathrm{mg} / \mathrm{dl}$. CKD was present in $47 \%$, and hypertension represented $61 \%$ of comorbidities. The proportion of patients with CKD in stages 2, 3, 4, and 5 were $7,40,18$, and $13 \%$, respectively. The median SAPS II score on admission to the ICU was 57 (range 44-72).

A summary of demographic data at the time of CRRT initiation is shown in Table 3. Mechanical ventilation, vasopressor, and diuretic use among patients was 79, 53, and 37\%, respectively. Renal data were as follows: serum creatinine at CRRT initiation was $2.7(1.9-4.0) \mathrm{mg} / \mathrm{dl}$, eGFR was $18(12-28) \mathrm{ml} / \mathrm{min} / 1.73 \mathrm{~m}^{2}$, urine output before CRRT initiation was $19(8-35) \mathrm{ml} / \mathrm{h}$, and BUN was $50(31-72) \mathrm{mg} / \mathrm{dl}$. Factors contributing to the development of AKI included sepsis (39\%), cardiogenic shock

Table 2 Baseline characteristics of the early and late CRRT groups

\begin{tabular}{|c|c|c|c|c|}
\hline & $\begin{array}{l}\text { All patients } \\
(n=189)\end{array}$ & $\begin{array}{l}\text { Early patients } \\
(n=52)\end{array}$ & $\begin{array}{l}\text { Late patients } \\
(n=137)\end{array}$ & $P$ value \\
\hline \multicolumn{5}{|l|}{ Baseline data } \\
\hline Age (years), median (IQR) & $72(61-78)$ & $74(62-78)$ & $71(61-77)$ & 0.37 \\
\hline Gender (male), $n$ (\%) & $133(70)$ & $36(69)$ & $97(71)$ & 0.86 \\
\hline Baseline serum creatinine $(\mathrm{mg} / \mathrm{dl})$, median (IQR) & $1.0(0.7-1.5)^{\mathrm{a}}$ & $1.5(0.9-2.9)^{\mathrm{a}}$ & $0.9(0.7-1.2)^{a}$ & $<0.001$ \\
\hline \multicolumn{5}{|l|}{ Comorbidities } \\
\hline Hypertension, $n$ (\%) & $116(61)$ & $36(69)$ & $80(58)$ & 0.19 \\
\hline Diabetes mellitus, n (\%) & $56(30)$ & $17(33)$ & $39(29)$ & 0.6 \\
\hline Chronic kidney disease, $n$ (\%) & $89(47)$ & $33(63)$ & $56(41)$ & 0.009 \\
\hline Stage $2, n(\%)$ & $6(7)$ & $2(4)$ & $4(3)$ & 0.69 \\
\hline Stage $3, n(\%)$ & $36(40)$ & $10(19)$ & $26(19)$ & 1 \\
\hline Stage $4, n(\%)$ & $16(18)$ & $10(19)$ & $6(4)$ & 0.002 \\
\hline Stage $5, n(\%)$ & $12(13)$ & $10(19)$ & $2(2)$ & $<0.001$ \\
\hline Unknown, n (\%) & $19(21)$ & $1(2)$ & 18(16) & 0.04 \\
\hline Congestive heart failure, $n(\%)$ & $34(18)$ & $10(19)$ & $24(18)$ & 0.83 \\
\hline \multicolumn{5}{|l|}{ Data on admission to ICU } \\
\hline Serum creatinine $(\mathrm{mg} / \mathrm{dl})$, median (IQR) & $2.0(1.2-3.4)$ & $1.5(1.1-2.9)$ & $2.1(1.3-3.5)$ & 0.047 \\
\hline Bilirubin (mg/dl), median (IQR) & $0.8(0.5-1.6)$ & $0.8(0.5-1.3)$ & $0.8(0.5-1.7)$ & 0.42 \\
\hline Platelets (cells/103), median (IQR) & $146(66-211)$ & $124(66-207)$ & $146(68-214)$ & 0.98 \\
\hline $\mathrm{PaO}_{2} / \mathrm{FiO}_{2}$ ratio (Torr), median (IQR) & $200(117-329)$ & $195(117-297)$ & $200(119-329)$ & 0.91 \\
\hline SAPS II, median (IQR) & $57(44-72)$ & $57(40-68)$ & $57(45-72)$ & 0.5 \\
\hline Glasgow Coma Scale, median (IQR) & $14(6-15)$ & $14(3-15)$ & $14(6-15)$ & 0.24 \\
\hline
\end{tabular}

Abbreviations: CRRT continuous renal replacement therapy, ICU intensive care unit, IQR interquartile range, BUN blood urea nitrogen, eGFR estimated glomerular filtration rate, SAPS // Simplified Acute Physiology Score II

${ }^{a}$ Sixty-five, six, and fifty-nine patients with unknown baseline creatinine were not included 
Table 3 Comparisons of demographic date at CRRT initiation and outcomes in early and late groups

\begin{tabular}{|c|c|c|c|c|}
\hline & $\begin{array}{l}\text { All patients } \\
(n=189)\end{array}$ & $\begin{array}{l}\text { Early group } \\
(n=52)\end{array}$ & $\begin{array}{l}\text { Late group } \\
(n=137)\end{array}$ & $P$ value \\
\hline \multicolumn{5}{|l|}{ Demographic data at CRRT initiation } \\
\hline Mechanical ventilation, $n(\%)$ & $150(79)$ & $45(87)$ & $105(77)$ & 0.16 \\
\hline Vasopressor, n (\%) & $101(53)$ & $26(50)$ & $75(55)$ & 0.63 \\
\hline Diuretic use, $n(\%)$ & $70(37)$ & $21(40)$ & $49(36)$ & 0.61 \\
\hline Creatinine $(\mathrm{mg} / \mathrm{dl})$, median (IQR) & $2.7(1.9-4.0)$ & $1.8(1.2-3.0)$ & $2.8(2.2-4.2)$ & $<0.001$ \\
\hline eGFR $\left(\mathrm{ml} / \mathrm{min} / 1.73 \mathrm{~m}^{2}\right)$ & $18(12-28)$ & $30(15-44)$ & $18(11-25)$ & $<0.001$ \\
\hline BUN (mg/dl), median (IQR) & $50(31-72)$ & $37(29-59)$ & $54(34-87)$ & $<0.001$ \\
\hline Urine Output (ml/h), median (IQR) & $19(8-35)$ & $21(10-46)$ & $17(7.6-33)$ & 0.09 \\
\hline Bilirubin (mg/dl), median (IQR) & $1.0(0.6-2.3)$ & $1.8(0.7-3.7)$ & $1.0(0.6-2.5)$ & 0.83 \\
\hline Platelets (cells/10³), median (IQR) & $112(63-188)$ & $107(65-159)$ & $118(62-194)$ & 0.84 \\
\hline Albumin (g/dl), median (IQR) & $2.5(2.0-3.1)$ & $2.5(2.1-3.1)$ & $2.5(2.0-3.1)$ & 0.87 \\
\hline $\mathrm{PiO}_{2} / \mathrm{FiO}_{2}$ (Torr), median (IQR) & $181(117-293)$ & $198(139-338)$ & $200(119-329)$ & 0.16 \\
\hline Glasgow Coma Scale, median (IQR) & $13(6-15)$ & $13(6-15)$ & $13(6-15)$ & 0.69 \\
\hline \multicolumn{5}{|l|}{ Factors contributing to AKI } \\
\hline Sepsis, $n(\%)$ & $74(39)$ & $21(40)$ & $53(39)$ & 0.87 \\
\hline Cardiogenic shock, n (\%) & $59(31)$ & $20(39)$ & $39(29)$ & 0.22 \\
\hline Drug-induced AKI, n (\%) & $4(2)$ & $1(2)$ & $3(2)$ & 1 \\
\hline Hypovolemia, n (\%) & $15(8)$ & $1(2)$ & $14(10)$ & 0.07 \\
\hline Post-operative, $n$ (\%) & $35(19)$ & $10(19)$ & $25(18)$ & 0.87 \\
\hline Post-renal failure, $n(\%)$ & $1(2)$ & $0(0)$ & $1(1)$ & 1 \\
\hline Others, $n(\%)$ & $7(4)$ & $2(4)$ & $5(4)$ & 1 \\
\hline \multicolumn{5}{|l|}{ Outcomes } \\
\hline Duration of CRRT (day), median (IQR) & $3(1-8)$ & $3(1-6)$ & $3(1-9)$ & 0.3 \\
\hline ICU length of stay (day), median (IQR) & $10(4-18)$ & $8(3-16)$ & $10(5-18)$ & 0.34 \\
\hline Post-CRRT hospital length of stay (day), median (IQR) & $11(1-32)$ & $13(1-32)$ & $11(1-32)$ & 0.81 \\
\hline Hospital length of stay (days), median, median (IQR) & $26(13-57)$ & $29(14-43)$ & $26(11-62)$ & 0.81 \\
\hline Time interval from ICU admission to CRRT initiation (day), median (IQR) & $0.8(0.1-1.9)$ & $0.8(0.2-2.0)$ & $0.8(0.1-1.9)$ & 0.89 \\
\hline ICU mortality, n (\%) & $86(47)$ & $26(50)$ & $60(44)$ & 0.51 \\
\hline Hospital mortality, $n$ (\%) & $101(53)$ & $33(64)$ & $68(50)$ & 0.1 \\
\hline
\end{tabular}

Others: hyperkalemia (two patients), acute pancreatitis, Goodpasture syndrome, mixed acidosis, acute renal stroke, and catastrophic anti-phospholipid syndrome Abbreviations: CRRT continuous renal replacement therapy, IQR interquartile range, BUN blood urea nitrogen, eGFR estimated glomerular filtration rate, AKI acute kidney injury, ICU intensive care unit

(31\%), drug-induced AKI (2\%), hypovolemia (8\%), postoperative (19\%), post-renal failure (2\%), and others (4\%). The "others" group included hyperkalemia (two patients), acute pancreatitis, Goodpasture syndrome, mixed acidosis, acute renal stroke, and catastrophic anti-phospholipid syndrome.

For the primary outcome, crude early vs. late group in-hospital mortality was 64 vs. $50 \%(P=0.10)$. For the secondary outcomes, the median CRRT length (3 vs. 3 days, $P=0.3$ ), the median post-CRRT hospital length of stay (13 vs. 11 days, $P=0.81$ ), the median hospital length of stay ( 29 vs. 26 days, $P=0.81$ ), the median ICU length of stay ( 8 vs. 8 days, $P=0.34$ ), the median time interval from ICU admission to CRRT initiation, and intensive care unit mortality (50 vs. $44 \%, P=0.51$ ) were not significant statistically.

As shown in Table 4, logistic regression analysis showed that late initiation of CRRT [odds ratio (OR), 0.30; 95\% confidence interval (CI), 0.13-0.71; $P=0.006]$ and lower SAPS II score (OR, 1.04; 95\% CI, 1.021.06; $P<0.001)$ were associated with lower in-hospital mortality.

\section{Discussion}

This is a single-center retrospective study evaluating the optimal timing for initiation of CRRT, as defined by the 
Table 4 Independent predictors of in-hospital mortality using logistic regression analysis

\begin{tabular}{llll}
\hline Variables & Odds ratio & $95 \%$ confidence interval & $P$ value \\
\hline \multicolumn{2}{l}{ Timing of RRT initiation } \\
Early initiation & $1.0^{\mathrm{a}}$ & & \\
Late initiation & 0.30 & $0.13-0.71$ & 0.006 \\
SAPS II score & 1.04 & $1.02-1.06$ & $<0.001$ \\
\hline
\end{tabular}

Abbreviations: RRT renal replacement therapy, SAPS // Simplified Acute Physiology Score II

${ }^{\mathrm{a}}$ Reference indicator variable

modified RIFLE classification in a mixed medical-surgical ICU. The major finding of this study is that late CRRT initiation, as defined by the modified RIFLE classification and lower SAPS scores, was associated with lower in-hospital mortality.

The optimal timing of RRT initiation for patients with AKI remains controversial because initiation of RRT can be influenced by the following factors: (1) patientspecific factors including renal function, co-morbid diseases, and primary diagnosis; (2) clinician-specific factors, including goals of therapy and local practice patterns; and (3) organizational factors including health costs, ICU type, and nursing availability [20]. Wellaccepted indications for initiating RRT in patients with AKI include volume overload, hyperkalemia, uremia, severe metabolic acidosis, and drug intoxication. However, since there is currently no consensus regarding the optimal timing of initiating RRT, some clinicians initiate RRT in patients with non-AKI or early-stage AKI to prevent the development of complications induced by AKI.

A previous report showed that earlier RRT initiation reduced mortality and contributed to better renal recovery [5]. A recent single-center randomized trial showed that early RRT initiation reduced the 90-day mortality compared with delayed RRT initiation (39.3 vs. 54.7\%, $P=0.003$ ) [6]. In this study, the early group consisted of patients within $8 \mathrm{~h}$ of diagnosis with stage 2 AKI in Kidney Disease Improving Global Outcome (KDIGO) criteria, while the late group consisted of patients after $12 \mathrm{~h}$ of diagnosis with stage 3 AKI. However, Bagshaw et al performed a prospective multicenter observational study at 54 centers in 23 countries enrolling 1238 patients, which showed that late initiation of RRT classified by creatinine was associated with lower mortality (OR, 0.46; 95\% CI, 0.36-0.58; $P<0.0001$ ) [21]. Another recent prospective multicenter randomized trial showed no apparent benefit from the early initiation strategy [7]. A recent systematic review and meta-analysis reported by Wierstra et al. concluded that early RRT initiation in critically ill patients with AKI did not result in a survival benefit [22]. A systematic review and meta-analysis of randomized controlled trials reported by $\mathrm{Xu}$ et al. showed similar results [23]. The data in the current study may support the results of previous studies, suggesting that early initiation strategy has no clinical benefit.

Differences in etiological background between the two groups including contributing factors to AKI and distribution of CKD deserve mention. First, in the early group, substantial numbers of patients might have had fluid overload, reflected by lower serum creatinine levels. In fact, studies suggest lower creatinine may reflect a state of fluid overload that can cause higher mortality $[24,25]$. Bouchard et al. conducted a prospective multicenter observational study including critically ill patients with AKI who needed RRT to assess whether fluid accumulation was associated with mortality with positive results (OR 2.07 [95\% CI 1.27-3.37]) and non-recovery of kidney function [26]. In our study, the number of patients with cardiogenic shock in the early group appeared larger than the late group (39 vs. 29\%). Those patients with cardiogenic shock may have well been complicated with fluid overload (Table 3). Physicians might not have recognized the true degree of AKI due to the apparent lower serum creatinine.

Second, more patients with CKD were included in the early group compared with the late group $(p=0.009)$. AKI in patients with CKD may be associated with a higher risk of mortality [27]. Wu et al. reported a multicenter observational study of 9425 patients for diagnosis of AKI after major surgery and evaluated mortality risk from post-operative AKI with or without CKD. The results showed that patients with a past history of CKD had higher mortality (HR, 3.94; 95\% CI, 2.79-5.28, $P<0.001)$ compared with the patients without CKD [28]. In this study, the mortality of patients with end-stage renal disease was higher than in patients with or without prior CKD. In the present study, there are fewer patients with CKD in the late RRT group than in the early RRT group. Also, the number of patients with CKD stages 4 and 5 were greater in the early RRT group than in the late RRT group (Table 2). For these hypothetical reasons, late RRT initiation was associated with a better prognosis in our study.

Finally, late RRT initiation policy has a potential to avoid unnecessary RRT. In a recent multicenter randomized controlled trial comparing the early vs. late policy, Gaudry et al. reported that renal function was more rapidly recovered and less frequency of catheter related blood stream infections occurred in late RRT initiation [7]. Patients with higher creatinine may undergo earlier treatment, and their prognosis would be better than that of patients with lower creatinine [29]. In our study, although there were no statistically significant differences regarding the time interval from ICU admission to RRT initiation in both early and late groups, more rapidly 
increasing creatinine appeared in the late group (Tables 2 and 3). It is possible that early treatment, including antibiotics, fluid challenges, nephrology consultation, and vasopressor use may have contributed to a better prognosis for patients with higher serum creatinine levels.

This study has several limitations. First, this study may have excluded a large number of AKI patients who met the criteria for RRT initiation but did not undergo CRRT. Second, this study was a single-center retrospective analysis with a relatively small sample size. Therefore, we cannot exclude the potential effects of unknown confounding factors. Third, we defined AKI according to the modified RIFLE classification and did not use urine criteria instead of the standard RIFLE classification. However, Haase et al. suggested that urine output criteria are not a more important predictive factor for in-hospital mortality than creatinine criteria [30]. Fourth, there was a lack of baseline creatinine data in approximately $34 \%$ of patients for whom we estimated the eGFR based on calculations. Therefore, the exact prevalence of CKD in the study population is unknown. Finally, some of the late group who started CRRT within $24 \mathrm{~h}$ of admission to ICU may have been early starters if laboratory testing had not been delayed, and there is potential for crossover of patients between the assigned groups.

\section{Conclusions}

The late initiation of CRRT, as defined by the modified RIFLE classification, is associated with a lower risk of inhospital mortality in critically ill patients with AKI. Further studies are needed to confirm the optimal timing for the initiation of CRRT.

\section{Abbreviations}

AKI: Acute kidney injury; BUN: Blood urea nitrogen; CKD: Chronic kidney disease; CRRT: Continuous renal replacement therapy; eGFR: Estimated glomerular filtration rate; HR: Hazard ratio; ICU: Intensive care unit; IQR: Interquartile range; KDIGO: Kidney Disease Improving Global Outcome; OR: Odds ratio; RRT: Renal replacement therapy; SAPS II: Simplified Acute Physiology Score II

\section{Acknowledgements}

All work was performed at the Jichi Medical University Saitama Medical Center. We thank Katsunobu Ando, Tsukasa Higuchi, and Makoto Hashimoto for clinical assistance. Part of this research was presented at the 26th Annual Meeting of the European Society of Intensive Care Medicine in Paris, 2013.

\section{Funding}

The authors declare that they have no funding for this study.

\section{Availability of data and materials}

The dataset supporting the conclusions of this article is included within the article.

\section{Authors' contributions}

All authors contributed the design of the study. JS participated in the data collection and drafted the manuscript. TO helped and revised the manuscript. HS, KI, TF, HY, AL, and MS participated in the data collection and manuscript writing. All authors read and approved the final manuscript.

\section{Competing interests}

The authors declare that they have no competing interests.

Consent for publication

Not applicable.

Ethics approval and consent to participate

This study was approved by the Institutional Review Board of Jichi Medical University Saitama Medical Center.

\section{Publisher's Note}

Springer Nature remains neutral with regard to jurisdictional claims in published maps and institutional affiliations.

\section{Author details \\ 'Division of Infectious Diseases, Jichi Medical University Hospital, 3311-1 Yakushiji, Shimotsuke, Tochigi 329-0498, Japan. ${ }^{2}$ Department of Anesthesiology and Critical Care Medicine, Jichi Medical University Saitama Medical Center, 1-847 Amanuma, Omiya-ku, Saitama City, Saitama 330-8503, Japan. ${ }^{3}$ Division of General Medicine, The First Department of Comprehensive Medicine, Jichi Medical University Saitama Medical Center, 1-847 Amanuma, Omiya-ku, Saitama City, Saitama 330-8503, Japan. ${ }^{4}$ Division of Nephrology, The First Department of Comprehensive Medicine, Jichi Medical University Saitama Medical Center, 1-847 Amanuma, Omiya-ku, Saitama City, Saitama 330-8503, Japan. ${ }^{5}$ Division of Cardiology, The First Department of Comprehensive Medicine, Jichi Medical University Saitama Medical Center, 1-847 Amanuma, Omiya-ku, Saitama City, Saitama 330-8503, Japan. ${ }^{6}$ Division of Endocrinology and Metabolism, The First Department of Comprehensive Medicine, Jichi Medical University Saitama Medical Center, \\ 1-847 Amanuma, Omiya-ku, Saitama City, Saitama 330-8503, Japan. \\ ${ }^{7}$ Department of Surgery, Jichi Medical University, 3311-1 Yakushiji, \\ Shimotsuke, Tochigi 329-0498, Japan.}

Received: 28 November 2016 Accepted: 8 April 2017

Published online: 10 July 2017

\section{Reference}

1. Ostermann M, Chang RW. Acute kidney injury in the intensive care unit according to RIFLE. Crit Care Med. 2007;35:1837-43.

2. Hoste EA, Clermont G, Kersten A, Venkataraman R, Angus DC, De Bacquer D, et al. RIFLE criteria for acute kidney injury are associated with hospital mortality in critically ill patients: a cohort analysis. Crit Care. 2006;10:R73.

3. Metnitz PG, Krenn CG, Steltzer H, Lang T, Ploder J, Lenz K, et al. Effect of acute renal failure requiring renal replacement therapy on outcome in critically ill patients. Crit Care Med. 2002;30:2051-8.

4. Uchino S, Kellum JA, Bellomo R, Doig GS, Morimatsu H, Morgera S, et al. Acute renal failure in critically ill patients: a multinational, multicenter study. JAMA. 2005;294:813-8.

5. Karvellas CJ, Farhat MR, Sajjad I, Mogensen SS, Leung AA, Wald R, et al. A comparison of early versus late initiation of renal replacement therapy in critically ill patients with acute kidney injury: a systematic review and meta-analysis. Crit Care. 2011;15:R72.

6. Zarbock A, Kellum JA, Schmidt C, Van Aken H, Wempe C, Pavenstädt H, et al. Effect of early vs delayed initiation of renal replacement therapy on mortality in critically ill patients with acute kidney injury: the ELAIN Randomized Clinical Trial. JAMA. 2016;315:2190-9.

7. Gaudry S, Hajage D, Schortgen F, Martin-Lefevre L, Pons B, Boulet E, et al. Initiation strategies for renal-replacement therapy in the intensive care unit. N Engl J Med. 2016;375:122-33.

8. Bellomo R, Ronco C, Kellum JA, Mehta RL, Palevsky P. Acute renal failure-definition, outcome measures, animal models, fluid therapy and information technology needs: the Second International Consensus Conference of the Acute Dialysis Quality Initiative (ADQI) Group. Crit Care. 2004;8:R204-12.

9. Bagshaw SM, George C, Bellomo R. A comparison of the RIFLE and AKIN criteria for acute kidney injury in critically ill patients. Nephrol Dial Transplant. 2008:23:1569-74.

10. Uchino S, Bellomo R, Goldsmith D, Bates S, Ronco C. An assessment of the RIFLE criteria for acute renal failure in hospitalized patients. Crit Care Med. 2006;34:1913-7. 
11. Shiao CC, Wu VC, Li WY, Lin YF, Hu FC, Young GH, et al. Late initiation of renal replacement therapy is associated with worse outcomes in acute kidney injury after major abdominal surgery. Crit Care. 2009;13:R171.

12. Shum HP, Chan KC, Kwan MC, Yeung AW, Cheung EW, Yan WW. Timing for initiation of continuous renal replacement therapy in patients with septic shock and acute kidney injury. Ther Apher Dial. 2013;17:305-10.

13. Wu SC, Fu CY, Lin HH, Chen RJ, Hsieh CH, Wang YC, et al. Late initiation of continuous veno-venous hemofiltration therapy is associated with a lower survival rate in surgical critically ill patients with postoperative acute kidney injury. Am Surg. 2012;78:235-42.

14. Chou YH, Huang TM, Wu VC, Wang CY, Shiao CC, Lai CF, et al. Impact of timing of renal replacement therapy initiation on outcome of septic acute kidney injury. Crit Care. 2011;15:R134.

15. National Kidney Foundation. K/DOQI clinical practice guidelines for chronic kidney disease: evaluation, classification, and stratification. Am J Kidney Dis. 2002;39:S1-26

16. Inker $L A$, Astor $B C$, Fox $C H$, Isakova T, Lash JP, Peralta CA, et al. KDOQI US commentary on the $2012 \mathrm{KDIGO}$ clinical practice guideline for the evaluation and management of CKD. Am J Kidney Dis. 2014;63:713-35.

17. Le Gall JR, Lemeshow S, Saulnier F. A new Simplified Acute Physiology Score (SAPS II) based on a European/North American multicenter study. JAMA. 1993:270:2957-63.

18. Levy MM, Fink MP, Marshall JC, Abraham E, Angus D, Cook D, et al. 2001 SCCM/ESICM/ACCP/ATS/SIS International Sepsis Definitions Conference. Crit Care Med. 2003;31:1250-6.

19. Kanda Y. Investigation of the freely available easy-to-use software "EZR" for medical statistics. Bone Marrow Transplant. 2013;48:452-8.

20. Vesconi S, Cruz DN, Fumagalli R, Kindgen-Milles D, Monti G, Marinho A, et al. Delivered dose of renal replacement therapy and mortality in critically ill patients with acute kidney injury. Crit Care. 2009;13:R57.

21. Bagshaw SM, Uchino S, Bellomo R, Morimatsu H, Morgera S, Schetz M, et al. Timing of renal replacement therapy and clinical outcomes in critically ill patients with severe acute kidney injury. J Crit Care. 2009;24:129-40.

22. Wierstra BT, Kadri S, Alomar S, Burbano X, Barrisford GW, Kao RLC. The impact of "early" versus "late" initiation of renal replacement therapy in critical care patients with acute kidney injury: a systematic review and evidence synthesis. Crit Care. 2016;20:122.

23. Xu Y, Gao J, Zheng X, Zhong B, Na Y, Wei J. Timing of initiation of renal replacement therapy for acute kidney injury: a systematic review and metaanalysis of randomized-controlled trials. Clin Exp Nephrol. 2016; Epub ahead of print.

24. Biesen Van W, Yegenaga I, Vanholder R, Verbeke F, Hoste E, Colardyn F, et al. Relationship between fluid status and its management on acute renal failure (ARF) in intensive care unit (ICU) patients with sepsis: a prospective analysis. J Nephrol. 2005;18(1):54-60.

25. Sakr Y, Vincent JL, Reinhart K, Groeneveld J, Michalopoulos A, Sprung CL, et al. High tidal volume and positive fluid balance are associated with worse outcome in acute lung injury. Chest. 2005:128:3098-108.

26. Bouchard J, Soroko SB, Chertow GM, Himmelfarb J, Ikizler TA, Paganini EP, et al. Fluid accumulation, survival and recovery of kidney function in critically ill patients with acute kidney injury. Kidney Int. 2009;76:422-7.

27. Rocha E, Soares M, Valente C, Nogueira L, Bonomo H, Godinho M, et al. Outcomes of critically ill patients with acute kidney injury and end-stage renal disease requiring renal replacement therapy: a case-control study. Nephrol Dial Transplant. 2009;24:1925-30.

28. Wu V-C, Huang T-M, Lai C-F, Shiao C-C, Lin Y-F, Chu T-S, et al. Acute-onchronic kidney injury at hospital discharge is associated with long-term dialysis and mortality. Kidney Int. 2011;80:1222-30.

29. Cerda J, Cerda M, Kilcullen P, Prendergast J. In severe acute kidney injury, a higher serum creatinine is paradoxically associated with better patient survival. Nephrol Dial Transpl. 2007:22:2781-4.

30. Haase M, Bellomo R, Matalanis G, Calzavacca P, Dragun D, Haase-Fielitz A. A comparison of the RIFLE and Acute Kidney Injury Network classifications for cardiac surgery-associated acute kidney injury: a prospective cohort study. J Thorac Cardiovasc Surg. 2009;138:1370-6.

\section{Submit your next manuscript to BioMed Central and we will help you at every step:}

- We accept pre-submission inquiries

- Our selector tool helps you to find the most relevant journal

- We provide round the clock customer support

- Convenient online submission

- Thorough peer review

- Inclusion in PubMed and all major indexing services

- Maximum visibility for your research

Submit your manuscript at www.biomedcentral.com/submit
Biomed Central 